\title{
Regularity for Ostwald-de Waele type shear thickening fluids
}

Hyeong-Ohk Bae, Kyungkeun Kang, Jihoon Lee and Jörg Wolf

\begin{abstract}
We obtain local in time existence of strong solution for nonNewtonian fluid with shear thickening viscosity. We also obtain the Hausdorff dimension of time singular set, and a Serrin type regularity criterion.
\end{abstract}

Mathematics Subject Classification (2010). 76D03, 76A05, 35Q35.

Keywords. Shear thickening fluid, Strong solution, Serrin criterion, Hausdorff dimension, Singularity, Regularity, Non-Newtonian fluid.

\section{Introduction. Statement of the main result}

We work on the existence of strong solutions for the incompressible fluid with shear thickening viscosity, especially, Ostwald-de Waele model (refer to [3]). Ladyzhenskaya first worked on such a flow in [14].

Let $\Omega \subset \mathbb{R}^{3}$ be the whole space $\Omega=\mathbb{R}^{3}$, or the periodic domain $\Omega \equiv$ $[0,1]^{3}$. Let $0<T<+\infty$. Set $Q_{T}:=\Omega \times(0, T)$. We consider a non-Newtonian incompressible fluid which is governed by the following system of PDE's

$$
\begin{aligned}
\operatorname{div} \mathbf{u} & =0 \quad \text { in } \quad Q_{T} \\
\mathbf{u}_{t}+(\mathbf{u} \cdot \nabla) \mathbf{u}-\operatorname{div} \sigma & =\mathbf{f}-\nabla p \quad \text { in } \quad Q_{T}
\end{aligned}
$$

Here, $\mathbf{u}=\left(u^{1}, u^{2}, u^{3}\right)^{\top}$ denotes the unknown velocity of the fluid and $p$ the pressure. Furthermore, $\mathbf{f}=\left(f^{1}, f^{2}, f^{3}\right)$ denotes a given external force. In addition, in the second equation $\sigma=\left(\sigma_{i j}\right)$ denotes the deviatoric stress which is defined by

$$
\sigma_{i j}=S_{i j}(\mathbf{D}(\mathbf{u})) \quad(i, j=1,2,3), \quad \mathbf{D}(\mathbf{u})=\frac{1}{2}\left(\nabla \mathbf{u}+(\nabla \mathbf{u})^{\top}\right) .
$$

Bae was partially supported by Basic Science Research Program through the National Research Foundation of Korea(NRF) funded by the Ministry of Education, Science and Technology (2009-0088692), and by World Class University project (R31-2009-000-20007-0). 
We impose the following condition on $\mathbf{S}=\left(S_{i j}\right)$ which is often called the deviatoric stress tensor.

$$
\begin{array}{rlrl}
S_{i j} & =S_{j i}, & & \text { (symmetric) } \\
\left|S_{i j}(\xi)\right| & \leq c_{1}|\xi|^{q-1}, \quad i, j=1,2,3, & & \text { (growth) } \\
\nu_{0}|\xi|^{q-2}|\eta|^{2} & \leq \frac{\partial S_{i j}}{\partial \xi_{k l}}(\xi) \eta_{k l} \eta_{i j} \leq \nu_{1}|\xi|^{q-2}|\eta|^{2}, & & \text { (coercivity) } \\
\exists H: \mathbf{M}_{\mathrm{sym}}^{3} \rightarrow \mathbb{R}: S_{i j}(\xi)=\frac{\partial H}{\partial \xi_{i j}}(\xi), & & \\
\nu_{2}|\xi|^{q} \leq H(\xi) \leq \nu_{3}|\xi|^{q} & & \text { (potential) }
\end{array}
$$

for all $\xi, \eta \in \mathbf{M}_{\text {sym }}^{3}$, where $\mathbf{M}_{\text {sym }}^{3}$ stands for the vector space of all symmetric $3 \times 3$ matrices.

We complete the system (1.1)-(1.2) with the initial condition

$$
\mathbf{u}=\mathbf{u}_{0} \quad \text { on } \Omega \times\{0\},
$$

and with the space periodic boundary condition when $\Omega=[0,1]^{3}$. When the periodic boundary condition is considered, we assume that the initial data and the external force have average zeros, so that the velocity is also average zero.

The existence of weak solutions is shown in $[14,15]$ with the periodic boundary condition, and in [18] in the whole space. Only recently, the existence of weak solutions with the Dirichlet boundary condition is shown in $[10,21]$. The existence of strong solutions is shown in [14] for $q \geq \frac{11}{5}$ with the periodic boundary condition. The strong solution is obtained for $q \geq \frac{9}{4}$ when the Laplacian term is added to $\sigma$ or $S$ in [16]. For $\frac{7}{5}<q<2$, the short time existence results of strong solutions with the periodic boundary condition are obtained in $[6,9]$. For $q=2$, the result is well known.

In this article, we have three directions. The first one is to show the short time existence of strong solutions in case of shear thickening fluids $q>2$. The second one is to obtain a Serrin type regularity criterion, and the last one is to obtain the Hausdorff dimension of the time singular set.

Serrin type regularity criteria for the Navier-Stokes flow $(q=2)$ have been studied by many researchers, for example, [11,19,20]. Later, the two component regularity criterion was studied in [7] for the vorticity. The two component regularity for the velocity was done in [1] in 1997, which was publishded in [2], and the one component regularity criterion in [17]. And there are more results in similar directions $[5,8,12,13,22]$, etc. For non-Newtonian fluids, such a regularity criterion is studied in [4] for shear thinning fluid.

Definition 1.1. Let $\mathbf{u}_{0} \in \mathbf{L}_{\sigma}^{2}(\Omega)$ and let $\mathbf{f} \in L^{q^{\prime}}\left(0, T ; \mathbf{W}^{-1, q^{\prime}}(\Omega)\right)$. We call $\mathbf{u} \in L^{\infty}\left(0, T ; \mathbf{L}_{\sigma}^{2}(\Omega)\right) \cap L^{q}\left(0, T ; \mathbf{W}_{0, \sigma}^{1, q}(\Omega)\right)$ a weak solution of $(1.1)-(1.7)$ with bounded energy, if 


$$
\begin{gathered}
\int_{Q_{T}}-\mathbf{u} \cdot \varphi_{t} d x d t-\int_{Q_{T}} \mathbf{u} \otimes \mathbf{u}: \nabla \varphi d x d t+\int_{Q_{T}} \mathbf{S}(\mathbf{D}(\mathbf{u})): \mathbf{D}(\varphi) d x d t \\
=\int_{0}^{T}\langle\mathbf{f}(t), \varphi(t)\rangle d t+\int_{\Omega} \mathbf{u}_{0} \cdot \varphi(0) d x
\end{gathered}
$$

for all $\varphi \in \mathbf{C}^{\infty}(\bar{Q})$ with $\operatorname{supp}(\varphi) \subset \Omega \times[0, T)$ and $\operatorname{div}_{x} \varphi=0$, and there holds the following energy inequality

$$
\frac{1}{2}\|\mathbf{u}(t)\|_{2}^{2}+\int_{0}^{t} \int_{\Omega} \sigma: \mathbf{D}(\mathbf{u}) d x d \tau \leq \frac{1}{2}\left\|\mathbf{u}_{0}\right\|_{2}^{2}+\int_{0}^{t} \int_{\Omega}\langle\mathbf{f}(\tau), \mathbf{u}(\tau)\rangle d \tau
$$

for almost all $t \in(0, T)$.

In the above, $q^{\prime}$ means the Hölder conjugate of $q$.

Definition 1.2. Let $\mathbf{u}_{0} \in \mathbf{W}^{1,2}(\Omega)$. We say that a weak solution $\mathbf{u}: Q_{T} \rightarrow \mathbb{R}^{3}$ is a strong solution to (1.1)-(1.7) if

$$
\begin{aligned}
& \nabla \mathbf{u} \in \mathbf{L}^{3}\left(Q_{T}\right) \cap L^{\infty}\left(0, T ; \mathbf{L}^{q} \cap \mathbf{L}^{2}(\Omega)\right), \\
& \mathbf{u}_{t} \in \mathbf{L}^{2}\left(Q_{T}\right), \quad \sigma \in L^{q^{\prime}}\left(0, T ; \mathbf{W}_{\mathrm{loc}}^{1, q^{\prime}}(\Omega)\right),
\end{aligned}
$$

and there holds

$$
\int_{Q_{T}}|\mathbf{D}(\mathbf{u})|^{q-2}|\nabla \mathbf{D}(\mathbf{u})|^{2} d x d t<+\infty .
$$

The aim of the present paper is to prove the short time existence of a strong solution to the system (1.1)-(1.7) in the following sense:

Theorem 1.3. Assume $q>2$. Let $\Omega$ be a periodic domain or the whole space. Let $\mathbf{u}_{0} \in \mathbf{W}^{1,2}(\Omega)$ be divergence free, $\nabla \mathbf{u}_{0} \in \mathbf{L}^{q}(\Omega), \mathbf{f} \in L^{2}\left(0, T ; \mathbf{L}^{2}(\Omega)\right)$ and $\nabla \mathbf{f} \in L^{3 / 2}\left(0, T ; \mathbf{L}^{3 / 2}(\Omega)\right)$. We also assume that $\mathbf{u}_{0}$ and $\mathbf{f}$ have average zero when $\Omega$ is a periodic domain. Then there exists $T_{0}$ with $0<T_{0} \leq T$ such that a weak solution $\mathbf{u}$ to (1.1)-(1.7) becomes strong in the cylinder $Q_{T_{0}}$.

The proof is provided in Sect. 2 .

The second and the third main results concern the regularity issue of weak solutions, which are provided in Theorems 1.4 and 1.5. If we consider the scaling invariance for the Eq. (1.2), the following scaling property of solutions is satisfied:

$$
\mathbf{u}_{\lambda}(x, t)=\lambda \mathbf{u}\left(\lambda^{\frac{3-q}{q-1}} x, \lambda^{\frac{2}{q-1}} t\right), \quad p_{\lambda}(x, t)=\lambda^{2} p\left(\lambda^{\frac{3-q}{q-1}} x, \lambda^{\frac{2}{q-1}} t\right) .
$$

Therefore, a Serrin's type condition for the gradient $\nabla \mathbf{u}$ is given as

$$
\nabla \mathbf{u} \in L_{t, x}^{\beta, \alpha} \equiv L^{\beta}\left(0, T ; \mathbf{L}^{\alpha}(\Omega)\right), \quad \text { where } \frac{3(3-q)}{\alpha}+\frac{2}{\beta}=2 .
$$

In the next theorem, we show that weak solutions become strong if the above condition is additionally assumed. 
Theorem 1.4. Let $2<q<11 / 5$. Suppose that $\mathbf{u}$ is a weak solution to (1.2) in $Q_{T}$. Assume further that $\nabla \mathbf{u} \in L_{t, x}^{\beta, \alpha}\left(Q_{T}\right)$ with $\frac{3(3-q)}{\alpha}+\frac{2}{\beta} \leq 2$ and $\alpha>\frac{3(3-q)}{2}$. Then $\mathbf{u}$ is a strong solution to (1.2) in $Q_{T}$. usual.

For $\alpha=\frac{3(3-q)}{2}$, for the regularity we need the smallness of the norm as

We estimate the Hausdorff dimension of the set of singular times.

Theorem 1.5. Let $2<q<11 / 5$. Let $\Omega$ be a periodic domain $[0,1]^{3}$ and $\mathbf{u}$ be a weak solution to $(1.1)-(1.7)$. Assume $\mathbf{f} \in L^{q^{\prime}}\left(0, T ; W^{1, q^{\prime}}(\Omega)\right)$. Then there exists a closed set $\mathcal{S} \subset[0, T]$, whose $\frac{2(11-5 q)}{22-15 q+3 q^{2}}$-dimensional Hausdorff measure vanishes, such that $\mathbf{u}$ is continuous from $[0, T] \backslash \mathcal{S}$ into $\mathbf{V}$.

Remark 1.6. (1) In [4], the Serrin type regularity condition and the Hausdorff dimensions of the set of the time singularity were obtained. More explicitly, the regularity condition for $\mathbf{u}$ is $\mathbf{u} \in L_{t, x}^{\beta, \alpha}\left(Q_{T}\right)$, where

$$
\frac{6}{\alpha}+\frac{5 q-6}{\beta} \leq 5 q-8 \quad \text { for } \frac{8}{5}<q<2,
$$

and the Hausdorff dimension of the time singularity was $\frac{12-5 q}{5 q-6}$ in [4].

(2) In [3] for $2<q<11 / 5$ the Hausdorff dimension of the time singular set was obtained as in Theorem 1.5, but for the proof the time independence of $\mathbf{f}$ was assumed, which should be fixed as we did in the proof of Theorem 1.5 in Sect. 3.

\section{Short time existence of strong solutions}

In this section we prove Theorem 1.3. To do that, we first consider an approximate system, and then, estimate the gradient of velocity.

\subsection{Approximate solutions}

In order to solve system (1.1)-(1.7) we proceed as in [10,21]. For given $\varepsilon>0$ we define

$$
\Phi_{\varepsilon}(\tau)=\Phi_{1}(\varepsilon \tau), \quad \tau \in \mathbb{R},
$$

where $\Phi_{1} \in C^{\infty}(\mathbb{R})$ denotes a cut-off function, such that $0 \leq \Phi_{1} \leq 1$ and $-2 \leq \Phi_{1}^{\prime} \leq 0$ in $\mathbb{R}, \Phi_{1} \equiv 1$ on $(-\infty, 1], \Phi_{1} \equiv 0$ in $(2,+\infty)$.

We use the notations $\|u\|_{\beta, \alpha ; Q_{T}}=\left(\int_{0}^{T}\left(\int_{\Omega}|u|^{\alpha} d x\right)^{\beta / \alpha} d t\right)^{1 / \beta}$, and $\|u\|_{\alpha ; Q_{T}}=\|u\|_{\alpha, \alpha ; Q_{T}}$.

By using the well-known monotone operator theory together with Banach's fixed point theorem one gets a weak solution $\mathbf{u}_{\varepsilon}$ with $\frac{\partial \mathbf{u}_{\varepsilon}}{\partial t} \in \mathbf{L}^{2}\left(Q_{T}\right)$ to the following approximate system

$$
\begin{aligned}
\operatorname{div} \mathbf{u}_{\varepsilon} & =0, \\
\mathbf{u}_{\varepsilon, t}+\left(\mathbf{u}_{\varepsilon} \Phi_{\varepsilon}\left(\left|\mathbf{u}_{\varepsilon}\right|\right) \cdot \nabla\right) \mathbf{u}_{\varepsilon}-\operatorname{div} \sigma_{\varepsilon} & =-\nabla p_{\varepsilon}+\mathbf{f} \quad \text { in } \quad Q_{T},
\end{aligned}
$$


together with initial condition (1.7), and also with the periodic boundary condition when $\Omega=[0,1]^{3}$. Here,

$$
\sigma_{\varepsilon}=\varepsilon \mathbf{D}\left(\mathbf{u}_{\varepsilon}\right)+\mathbf{S}\left(\mathbf{D}\left(\mathbf{u}_{\varepsilon}\right)\right) \text { a. e. in } Q_{T} .
$$

Taking into account (1.4) and (1.5) together with Korn's inequality we obtain the a priori estimate

$$
\left\|\mathbf{u}_{\varepsilon}\right\|_{L^{\infty}\left(0, T ; \mathbf{L}^{2}\right)}^{2}+\left\|\nabla \mathbf{u}_{\varepsilon}\right\|_{q}^{q} \leq c\left\|\mathbf{u}_{0}\right\|_{2}^{2}+c\|\mathbf{f}\|_{2}^{2}
$$

By means of reflexivity and a standard compactness argument we can choose a sequence $\left(\varepsilon_{m}\right)$ such that $\varepsilon_{m} \rightarrow 0$ and

$$
\begin{aligned}
& \mathbf{u}_{\varepsilon_{m}} \rightarrow \mathbf{u} \quad \text { in } \quad L^{q}\left(0, T ; \mathbf{W}^{1, q}(\Omega)\right), \\
& \mathbf{u}_{\varepsilon_{m}} \stackrel{*}{\rightarrow} \mathbf{u} \quad \text { in } \quad L^{\infty}\left(0, T ; \mathbf{L}^{2}(\Omega)\right), \\
& \mathbf{u}_{\varepsilon_{m}} \rightarrow \mathbf{u} \quad \text { in } \quad \mathbf{L}^{2}\left(Q_{T}\right)
\end{aligned}
$$

As it has been proved in $[16,21], \mathbf{u}$ is a weak solution to (1.1)-(1.7) with bounded energy.

(2). In view of (1.6) we have

$$
\begin{aligned}
\int_{Q_{t}} \mathbf{S}\left(\mathbf{D}\left(u_{\varepsilon}\right)\right): \mathbf{D}\left(u_{\varepsilon, t}\right) d x d \tau & =\int_{Q_{t}} \frac{\partial H}{\partial \xi_{i j}}\left(\mathbf{D}\left(u_{\varepsilon}\right)\right) D_{i j}\left(u_{\varepsilon, t}\right) d x d \tau \\
& =\int_{0}^{t} \frac{d}{d t} \int_{\Omega} H\left(\mathbf{D}\left(u_{\varepsilon}\right)\right) d x d \tau \\
& =\int_{\Omega} H\left(\mathbf{D}\left(\mathbf{u}_{\varepsilon}(t)\right)\right) d x-\int_{\Omega} H\left(\mathbf{D}\left(\mathbf{u}_{0}\right)\right) d x
\end{aligned}
$$

Once more making use of (1.6) this shows that ${ }^{1}$

$$
\begin{aligned}
\left\|\mathbf{u}_{\varepsilon, t}\right\|_{2 ; Q_{T^{\prime}}}^{2} & +\left\|\nabla \mathbf{u}_{\varepsilon}\right\|_{L^{\infty}\left(0, T^{\prime} ; \mathbf{L}^{q}(\Omega)\right)}^{q} \\
& \leq c\left\|\nabla \mathbf{u}_{0}\right\|_{q}^{q}+c\left\|\left(\mathbf{u}_{\varepsilon} \Phi_{\varepsilon}\left(\left|\mathbf{u}_{\varepsilon}\right|\right) \cdot \nabla\right) \mathbf{u}_{\varepsilon}\right\|_{2 ; Q_{T^{\prime}}}^{2}+\|\mathbf{f}\|_{2 ; Q_{T^{\prime}}}^{2} \\
& \leq c \mathcal{K}^{2}+c \int_{Q_{T^{\prime}}}\left|\mathbf{u}_{\varepsilon}\right|^{2}\left|\nabla \mathbf{u}_{\varepsilon}\right|^{2} d x d t
\end{aligned}
$$

where

$$
\mathcal{K}=\left\|\nabla \mathbf{u}_{0}\right\|_{q}^{q / 2}+\left\|\nabla \mathbf{u}_{0}\right\|_{2}+\|\mathbf{f}\|_{2 ; Q_{T^{\prime}}} .
$$

for every $0<T^{\prime} \leq T$.

Since for $q \geq \frac{11}{5}$, the regularity of solutions are known, we restrict the case $2<q<\frac{11}{5}$.

\footnotetext{
${ }^{1}$ Remark, by the definition of $\Phi_{\varepsilon}$ we have $\mathbf{u}_{\varepsilon} \Phi_{\varepsilon}\left(\left|\mathbf{u}_{\varepsilon}\right|\right) \leq \frac{2}{\varepsilon}$. Hence, owing to $\nabla \mathbf{u}_{\varepsilon} \in \mathbf{L}^{q}\left(Q_{T}\right)$ there holds $\left(\mathbf{u}_{\varepsilon} \Phi_{\varepsilon}\left(\left|\mathbf{u}_{\varepsilon}\right|\right) \cdot \nabla\right) \mathbf{u}_{\varepsilon} \in \mathbf{L}^{2}\left(Q_{T}\right)$.
} 
Lemma 2.1. Let $\mathbf{u}_{\varepsilon}$ be a solution to the approximate system. Then there holds

$$
\begin{aligned}
\left\|\mathbf{u}_{\varepsilon, t}\right\|_{2 ; Q_{T^{\prime}}}^{2} & +\left\|\nabla \mathbf{u}_{\varepsilon}\right\|_{\infty, q ; Q_{T^{\prime}}}^{q}+\left\|\left|\mathbf{u}_{\varepsilon}\left\|\nabla \mathbf{u}_{\varepsilon} \mid\right\|_{2 ; Q_{T^{\prime}}}^{2}\right.\right. \\
& \leq c\left(\mathcal{K}^{2}+\mathcal{K}^{4 q \rho}\left(T^{\prime}\right)^{(7 q-12) v}\|\nabla \mathbf{u}\|_{q, 3 q ; Q_{T^{\prime}}}^{3 q(4-q) v}\right),
\end{aligned}
$$

where $\rho=\frac{7 q-12}{(5 q+12)(q-2)}$ and $v=\frac{5 q-6}{(5 q+12)(q-2)}$.

Proof. For the sake of notational simplicity we omit the subscript $\varepsilon$ and write $\mathbf{u}$ instead of $\mathbf{u}_{\varepsilon}$.

Using Cauchy-Schwarz's inequality, we get

$$
\int_{Q_{T^{\prime}}}|\mathbf{u}|^{2}|\nabla \mathbf{u}|^{2} d x d t \leq\|\mathbf{u}\|_{\infty, 4 ; Q_{T^{\prime}}}^{2}\|\nabla \mathbf{u}\|_{2,4 ; Q_{T^{\prime}}}^{2} .
$$

With help of Hölder's inequality along with Sobolev's embedding theorem we find

$$
\|\mathbf{u}(t)\|_{4 ; \Omega}^{2} \leq c\|\mathbf{u}(t)\|_{2 ; \Omega}^{\frac{7 q-12}{5 q-6}}\|\nabla \mathbf{u}(t)\|_{q ; \Omega}^{\frac{3 q}{5 q-6}} \quad \text { a.e. } t \in(0, T) .
$$

Thus, the former inequality with the aid of the latter inequality implies

$$
\int_{Q_{T^{\prime}}}|\mathbf{u}|^{2}|\nabla \mathbf{u}|^{2} d x d t \leq c\|\mathbf{u}\|_{\infty, 2 ; Q_{T}}^{\frac{7 q-12}{5 q-6}}\|\nabla \mathbf{u}\|_{\infty, q ; Q_{T^{\prime}}}^{\frac{3 q}{5 q-6}}\|\nabla \mathbf{u}\|_{2,4 ; Q_{T^{\prime}}}^{2} .
$$

Then, estimating the right hand side by the aid of (2.2) and (2.6) we get

$$
\int_{Q_{T^{\prime}}}|\mathbf{u}|^{2}|\nabla \mathbf{u}|^{2} d x d t \leq c \mathcal{K}^{\frac{7 q-12}{5 q-6}}\left(\mathcal{K}^{2}+\int|u|^{2}|\nabla u|^{2}\right)^{\frac{3}{5 q-6}}\|\nabla \mathbf{u}\|_{2,4 ; Q_{T^{\prime}}}^{2},
$$

so, using Young's inequality we get

$$
\int_{Q_{T^{\prime}}}|\mathbf{u}|^{2}|\nabla \mathbf{u}|^{2} d x d t \leq c\left(\mathcal{K}^{2}+\mathcal{K}^{\frac{7 q-12}{5 q-9}}\|\nabla \mathbf{u}\|_{2,4 ; Q_{T^{\prime}}}^{\frac{10 q-12}{5 q-9}}\right) .
$$

For convenience, we denote

$$
\mu=\frac{7 q-12}{5 q-9}, \quad \eta=\frac{5 q-6}{5 q-9} .
$$

Let $\theta \in(0,1)$ and $s \in(1,2)$ such that

$$
\frac{1-\theta}{q}+\frac{\theta}{3 q}=\frac{1}{4}, \quad \frac{1-\theta}{s}+\frac{\theta}{q}=\frac{1}{2} .
$$

Clearly,

$$
\theta=\frac{12-3 q}{8}, \quad 1-\theta=\frac{3 q-4}{8}, \quad s=\frac{q(3 q-4)}{7 q-12} .
$$

By means of Hölder's inequality we get

$$
\|\nabla \mathbf{u}\|_{2,4 ; Q_{T^{\prime}}} \leq\|\nabla \mathbf{u}\|_{s, q ; Q_{T^{\prime}}}^{1-\theta}\|\nabla \mathbf{u}\|_{q, 3 q ; Q_{T^{\prime}}}^{\theta} \leq\left(T^{\prime}\right)^{\frac{1-\theta}{s}}\|\nabla \mathbf{u}\|_{\infty, q ; Q_{T^{\prime}}}^{1-\theta}\|\nabla \mathbf{u}\|_{q, 3 q ; Q_{T^{\prime}}}^{\theta} .
$$

In view of $\frac{1-\theta}{s}=\frac{1}{2}-\frac{\theta}{q}=\frac{7 q-12}{8 q}$ we see that

$$
\|\nabla \mathbf{u}\|_{2,4 ; Q_{T^{\prime}}} \leq\left(T^{\prime}\right)^{\frac{7 q-12}{8 q}}\|\nabla \mathbf{u}\|_{\infty, q ; Q_{T^{\prime}}}^{\frac{3 q-4}{8}}\|\nabla \mathbf{u}\|_{q, 3 q ; Q_{T^{\prime}}}^{\frac{12-3 q}{8}} .
$$


Inserting this inequality into (2.8) we get

$$
\int_{Q_{T^{\prime}}}|\mathbf{u}|^{2}|\nabla \mathbf{u}|^{2} d x d t \leq c\left(\mathcal{K}^{2}+\mathcal{K}^{\mu}\left(T^{\prime}\right)^{\frac{\mu(5 q-6)}{4 q}}\|\nabla \mathbf{u}\|_{\infty, q ; Q_{T^{\prime}}}^{\frac{\eta(3 q-4)}{4}}\|\nabla \mathbf{u}\|_{q, 3 q ; Q_{T^{\prime}}}^{\frac{\eta(12-3 q)}{4}}\right) .
$$

By using (2.6) and Young's inequality we are led to

$$
\begin{aligned}
& \int_{Q_{T^{\prime}}}|\mathbf{u}|^{2}|\nabla \mathbf{u}|^{2} d x d t \\
& \left.\leq c\left(\mathcal{K}^{2}+\mathcal{K}^{\mu}\left(T^{\prime}\right)^{\frac{\mu(5 q-6)}{4 q}}\left[\mathcal{K}^{2}+\||u||\nabla u|\|_{2 ; Q_{T^{\prime}}}^{2}\right]^{\frac{\eta(3 q-4)}{4 q}}\right]\|\nabla \mathbf{u}\|_{q, 3 q ; Q_{T^{\prime}}}^{\frac{\eta(12-3 q)}{4}}\right) \\
& \leq c\left(\mathcal{K}^{2}+\mathcal{K}^{\frac{4 q(7 q-12)}{(5 q+12)(q-2)}}\left(T^{\prime}\right)^{\frac{(7 q-12)(5 q-6)}{(5 q+12)(q-2)}}\|\nabla \mathbf{u}\|_{q, 3 q ; Q_{T^{\prime}}}^{\frac{q(12-3 q)(5 q-6)}{(5 q+2)(q-2)}}\right)+\frac{1}{2}\|\mid u\| \nabla u \|_{2 ; Q_{T^{\prime}}}^{2},
\end{aligned}
$$

therefore,

$$
\int_{Q_{T^{\prime}}}|\mathbf{u}|^{2}|\nabla \mathbf{u}|^{2} d x d t \leq c\left(\mathcal{K}^{2}+\mathcal{K}^{\frac{4 q(7 q-12)}{(5 q+12)(q-2)}}\left(T^{\prime}\right)^{\frac{(7 q-12)(5 q-6)}{(5 q+12)(q-2)}}\|\nabla \mathbf{u}\|_{q, 3 q ; Q_{T^{\prime}}}^{\frac{q(12-3 q)(5 q-6)}{(5 q+12)(q-2)}}\right) .
$$

Finally, (2.7) follows from the last inequality by using (2.2).

\subsection{Gradient estimates of stress of the approximate solution}

The aim of this section is to provide some important gradient estimates for the stress $\sigma_{\varepsilon}=\mathbf{S}\left(\mathbf{D}\left(\mathbf{u}_{\varepsilon}\right)\right)$, where $\mathbf{u}_{\varepsilon}$ denotes the solution of the approximate system (2.1). In order to simplify notations throughout this section, instead of $\mathbf{u}_{\varepsilon}, p_{\varepsilon}, \sigma_{\varepsilon}$, etc., we will write $\mathbf{u}, p, \sigma$.

Since $(\mathbf{u}, p)$ is a weak solution to $(2.1)$ and $\mathbf{u}_{t} \in \mathbf{L}^{2}\left(Q_{T}\right)$, we see that $(\mathbf{u}, p)$ satisfies the following integral identity

$$
\begin{aligned}
& \int_{0}^{T^{\prime}} \quad \int_{\Omega} \mathbf{u}_{t} \cdot \varphi d y d t+\int_{0}^{T^{\prime}} \int_{\Omega} \sigma: \mathbf{D} \varphi d y d t \\
& \quad=-\int_{0}^{T^{\prime}} \int_{\Omega} \mathbf{h}_{\varepsilon}: \varphi d y d t+\int_{0}^{T^{\prime}} \int_{\Omega} p \operatorname{div} \varphi d y d t+\int_{0}^{T^{\prime}} \int_{\Omega} \mathbf{f} \cdot \varphi d y d t
\end{aligned}
$$

for all $0<T^{\prime} \leq T$ and for all $\varphi \in \mathbf{C}^{\infty}\left(\mathbb{R}, \mathbf{C}_{0}^{\infty}\left(\mathbb{R}^{3}\right)\right)$, or for all $\varphi \in \mathbf{C}^{\infty}\left(\mathbb{R}^{4}\right)$ with the spatial periodic boundary condition if $\Omega=[0,1]^{3}$, where

$$
\mathbf{h}_{\varepsilon}=\nabla \mathbf{u} \cdot \mathbf{u} \Phi_{\varepsilon}(|\mathbf{u}|) \quad \text { a.e. in } \quad Q_{T} .
$$

Let $\gamma \in\{1,2,3\}$. By $\mathbf{e}_{\gamma}$ we denote the unit vector $(1,0,0),(0,1,0),(0,0,1)$ for $\gamma=1,2,3$, respectively. Let $\Delta^{h} \varphi$ denote the difference quotient

$$
\Delta^{h} \varphi(y)=\frac{\varphi\left(y+h \mathbf{e}_{\gamma}\right)-\varphi(y)}{h}, \quad y \in \mathbb{R}^{3}
$$


Then, into (2.9) we inserting $\varphi=-\Delta^{-h} \Delta^{h} \mathbf{u}$, applying integration by parts we get

$$
\begin{aligned}
& \frac{1}{2}\left\|\Delta^{h} \mathbf{u}\left(T^{\prime}\right)\right\|_{2 ; \Omega}^{2}+\int_{Q_{T^{\prime}}} \Delta^{h} \sigma: \Delta^{h} \mathbf{D} \mathbf{u} d y d t \\
& =\frac{1}{2}\left\|\Delta^{h} \mathbf{u}(0)\right\|_{2 ; \Omega}^{2}-\int_{Q_{T^{\prime}}} \Delta^{h} \mathbf{h}_{\varepsilon}: \Delta^{h} \mathbf{u} d y d t+\int_{Q_{T^{\prime}}} \Delta^{h} \mathbf{f} \cdot \Delta^{h} \mathbf{u} d y d t
\end{aligned}
$$

Estimating the right hand side of (2.10) we are led to

$$
\begin{aligned}
\int_{Q_{T^{\prime}}} & \Delta^{h} \sigma: \Delta^{h} \mathbf{D} \mathbf{u} d y d t \leq \frac{1}{2}\left\|\Delta^{h} \mathbf{u}(0) \zeta_{0}\right\|_{2}^{2} \\
+ & \left|\int_{Q_{T^{\prime}}} \Delta^{h} \mathbf{h}_{\varepsilon}:\left(\Delta^{h} \mathbf{u}\right) \zeta_{0}^{2} d y d t\right|+\left|\int_{Q_{T^{\prime}}} \Delta^{h} \mathbf{f} \cdot \Delta^{h} \mathbf{u} d y d t\right|
\end{aligned}
$$

We recall the product rules for the difference quotients.

$$
\begin{aligned}
& \Delta^{h}(f g)=\Delta^{h} f g+f\left(\cdot+h \mathbf{e}_{\gamma}\right) \Delta^{h} g, \\
& \Delta^{-h}(f g)=\Delta^{-h} f g\left(\cdot-h \mathbf{e}_{\gamma}\right)+f \Delta^{-h} g, \text { and } \\
& \left(\Delta^{-h} f\right)\left(\cdot+h \mathbf{e}_{\gamma}\right)=\Delta^{h} f .
\end{aligned}
$$

Now, by the fundamental theorem of calculus we calculate

$$
\Delta^{h} \sigma=\int_{0}^{1} \frac{\partial \mathbf{S}}{\partial \xi_{k l}}\left(\mathbf{D}(\mathbf{u})+\tau h \Delta^{h} \mathbf{D}(\mathbf{u})\right) d \tau \Delta^{h} D_{k l}(\mathbf{u}) \quad \text { a.e. in } \quad Q_{T}
$$

Observing (1.5) we see that

$$
\begin{aligned}
\Delta^{h} & \sigma: \Delta^{h} \mathbf{D}(\mathbf{u}) \\
& =\int_{0}^{1} \frac{\partial S_{i j}}{\partial \xi_{k l}}\left(\mathbf{D}(\mathbf{u})+\tau h \Delta^{h} \mathbf{D}(\mathbf{u})\right) d \tau \Delta^{h} D_{k l}(\mathbf{u}) \Delta^{h} D_{i j}(\mathbf{u}) \\
& \geq \nu_{0} \int_{0}^{1}\left|(1-\tau) \mathbf{D}(\mathbf{u})+\tau \mathbf{D}\left(\mathbf{u}\left(\cdot+h \mathbf{e}_{\gamma}\right)\right)\right|^{q-2} d \tau\left|\Delta^{h} \mathbf{D}(\mathbf{u})\right|^{2}+\varepsilon\left|\Delta^{h} \mathbf{D}(\mathbf{u})\right|^{2} \\
& \geq 2^{-q-2} \nu_{0}\left|\mathbf{D}(\mathbf{u})+\mathbf{D}\left(\mathbf{u}\left(\cdot+h \mathbf{e}_{\gamma}\right)\right)\right|^{q-2}\left|\Delta^{h} \mathbf{D}(\mathbf{u})\right|^{2}+\varepsilon\left|\Delta^{h} \mathbf{D}(\mathbf{u})\right|^{2} .
\end{aligned}
$$

The inequality above together with (2.11) yields

$$
\begin{aligned}
& \int_{Q_{T^{\prime}}}\left(\varepsilon+\left|\mathbf{D}(\mathbf{u})+\mathbf{D}\left(\mathbf{u}\left(\cdot+h \mathbf{e}_{\gamma}\right)\right)\right|^{q-2}\right)\left|\Delta^{h} \mathbf{D}(\mathbf{u})\right|^{2} d y d t \\
& \quad \leq c\left\|\Delta^{h} \mathbf{u}(0)\right\|_{2}^{2}+c\left|\int_{Q_{T^{\prime}}} \Delta^{h} \mathbf{h}_{\varepsilon}: \Delta^{h} \mathbf{u} d y d t\right|+c\left|\int_{Q_{T^{\prime}}} \Delta^{h} \mathbf{f} \cdot \Delta^{h} \mathbf{u} d y d t\right| .
\end{aligned}
$$

On the other hand, from (2.13) along with (1.5) we estimate

$$
\left|\Delta^{h} \sigma\right| \leq 2^{q} \nu_{1}\left(\varepsilon+\left|\mathbf{D}(\mathbf{u})+\mathbf{D}\left(\mathbf{u}\left(\cdot+h \mathbf{e}_{\gamma}\right)\right)\right|^{q-2}\right)\left|\Delta^{h} \mathbf{D}(\mathbf{u})\right| \quad \text { a.e. in } \quad Q_{T} \text {. }
$$


With help of Hölder's inequality we find

$$
\begin{aligned}
\left\|\Delta^{h} \sigma\right\|_{q^{\prime} ; Q_{T^{\prime}}}^{2} & \leq c\left(\int_{Q_{T^{\prime}}}\left(\varepsilon+\left|\mathbf{D}(\mathbf{u})+\mathbf{D}\left(\mathbf{u}\left(\cdot+h \mathbf{e}_{\gamma}\right)\right)\right|^{(q-2)}\right)^{q^{\prime}}\left|\Delta^{h} \mathbf{D}(\mathbf{u})\right|^{q^{\prime}} d y d t\right)^{\frac{2}{q^{\prime}}} \\
& \leq \mathcal{K}_{\varepsilon}^{\frac{2(q-2)}{q}} \int_{Q_{T^{\prime}}}\left(\varepsilon+\left|\mathbf{D}(\mathbf{u})+\mathbf{D}\left(\mathbf{u}\left(\cdot+h \mathbf{e}_{\gamma}\right)\right)\right|^{q-2}\right)\left|\Delta^{h} \mathbf{D}(\mathbf{u})\right|^{2} d y d t
\end{aligned}
$$

where

$$
\mathcal{K}_{\varepsilon}=c \varepsilon^{\frac{q}{q-2}}+\mathcal{K}
$$

Since $\mathcal{K}_{\varepsilon}>0$ without loss of generality we may assume that $\varepsilon^{\frac{q}{q-2}} \leq\left\|\mathbf{u}_{0}\right\|_{2} \leq \mathcal{K}$. Therefore, in what follows we may replace $\mathcal{K}_{\varepsilon}$ by $\mathcal{K}$. Combining this inequality with (2.14) and using Young's inequality one obtains

$$
\begin{aligned}
\left\|\Delta^{h} \sigma\right\|_{q^{\prime} ; Q_{T^{\prime}}}^{2} \leq & \mathcal{K}^{\frac{2(q-2)}{q}}\left\|\nabla \mathbf{u}_{0}\right\|_{2}^{2}+\mathcal{K}^{\frac{2(q-2)}{q}}\left|\int_{Q_{T^{\prime}}} \Delta^{h} \mathbf{h}_{\varepsilon}: \Delta^{h} \mathbf{u} d y d t\right| \\
& +\mathcal{K}^{\frac{2(q-2)}{q}}\left|\int_{Q_{T^{\prime}}} \Delta^{h} \mathbf{f}: \Delta^{h} \mathbf{u} d y d t\right|
\end{aligned}
$$

In order to verify the regularity of $\mathbf{h}_{\varepsilon}$ we first mention that $\nabla \mathbf{u} \in$ $L^{\infty}\left(0, T ; \mathbf{L}^{q}(\Omega)\right)$ (owing to (2.6) and its related remark) and $\mathbf{u} \in L^{2}\left(0, T ; \mathbf{W}^{2,2}\right.$ $(\Omega))$ along with Sobolev's embedding theorem imply that $\nabla \mathbf{u} \in \mathbf{L}^{\frac{6+2 q}{3}}\left(Q_{T}\right) \hookrightarrow$ $\mathbf{L}^{3}\left(Q_{T}\right)$. In addition, using Hölder's inequality we see that $\nabla \mathbf{u} \in L^{6}\left(0, T ; \mathbf{L}^{\frac{18 q}{12+q}}\right.$ $(\Omega)) \hookrightarrow L^{6}\left(0, T ; \mathbf{L}^{2}(\Omega)\right)$. By means of Sobolev's embedding theorem we get $\mathbf{u} \in \mathbf{L}^{6}\left(Q_{T}\right)$. This shows that

$$
\left|\nabla \mathbf{h}_{\varepsilon}\right| \leq c|\nabla \mathbf{u}|^{2}+c|\mathbf{u}|\left|D^{2} \mathbf{u}\right| \in \mathbf{L}^{\frac{3}{2}}\left(Q_{T}\right) .
$$

Now, by means of reflexivity and Riesz-Fischer's theorem we may pass to the limit $h \rightarrow 0$ on both sides of (2.14) and (2.15) to obtain

$$
\left\|\sigma_{y_{\gamma}}\right\|_{q^{\prime} ; Q_{T^{\prime}}}^{2} \leq \mathcal{K}^{\frac{2(q-2)}{q}}\left(\mathcal{K}^{2}+\left|\int_{Q_{T^{\prime}}} \mathbf{h}_{\varepsilon, y_{\gamma}}: \mathbf{u}_{y_{\gamma}} d y d t\right|+\left|\int_{Q_{T^{\prime}}} \mathbf{f}_{y_{\gamma}}: \mathbf{u}_{y_{\gamma}} d y d t\right|\right)
$$

and

$$
\begin{aligned}
\int_{Q_{T^{\prime}}}|\mathbf{D}(\mathbf{u})|^{q-2}\left|(\mathbf{D}(\mathbf{u}))_{y_{\gamma}}\right|^{2} d y d t \leq & \mathcal{K}^{2}+\left|\int_{Q_{T^{\prime}}} \mathbf{h}_{\varepsilon, y_{\gamma}}: \mathbf{u}_{y_{\gamma}} d y d t\right| \\
& +\left|\int_{Q_{T^{\prime}}} \mathbf{f}_{y_{\gamma}} \cdot \mathbf{u}_{y_{\gamma}} d y d t\right|
\end{aligned}
$$

Finally, it remains to estimate the second integral on the right of (2.16) and (2.17), respectively. For, we make use of the transformation formula of the Lebesgue integral and get

$$
\int_{Q_{T^{\prime}}} \mathbf{h}_{\varepsilon, y_{\gamma}}: \mathbf{u}_{y_{\gamma}} d y d t=\int_{Q_{T^{\prime}}}\left(u_{x_{j}}^{i} u^{j} \Phi_{\varepsilon}(|\mathbf{u}|)\right)_{x_{\gamma}} u_{x_{\gamma}}^{i} d x d t
$$


Calculating

$$
\left(u_{x_{j}}^{i} u^{j} \Phi_{\varepsilon}(|\mathbf{u}|)\right)_{x_{\gamma}}=\left(u_{x_{\gamma}}^{i}\right)_{x_{j}} u^{j} \Phi_{\varepsilon}(|\mathbf{u}|)+u_{x_{j}}^{i}\left(u^{j} \Phi_{\varepsilon}(|\mathbf{u}|)\right)_{x_{\gamma}}
$$

from (2.18) using integration by parts together with the transformation formula of the Lebesgue integral we deduce

$$
\begin{aligned}
\int_{Q_{T^{\prime}}} \mathbf{h}_{\varepsilon, y_{\gamma}}: \mathbf{u}_{y_{\gamma}} d y d t= & -\frac{1}{2} \int_{Q_{T^{\prime}}}\left|\mathbf{u}_{y_{\gamma}}\right|^{2} u^{j}\left(\Phi_{\varepsilon}(|\mathbf{u}|)\right)_{y_{j}} d y d t \\
& +\int_{Q_{T^{\prime}}} u_{y_{j}}^{i} u_{y_{\gamma}}^{i}\left(u^{j} \Phi_{\varepsilon}(|\mathbf{u}|)\right)_{y_{\gamma}} d y d t
\end{aligned}
$$

From this identity and the inequality

$$
\left|\left(u^{j} \Phi_{\varepsilon}(|\mathbf{u}|)\right)_{y_{k}}\right| \leq c|\nabla \mathbf{u}| \quad \text { a.e. in } \quad U^{+} \times(0, T),
$$

we easily see that

$$
\begin{aligned}
\left|\int_{0}^{T^{\prime}} \int_{\Omega} \mathbf{h}_{\varepsilon, y_{\gamma}}: \mathbf{u}_{y_{\gamma}} d y d t\right| & \leq c \int_{Q_{T^{\prime}}}|\nabla \mathbf{u}|^{3} d x d t+c \int_{Q_{T^{\prime}}}|\nabla \mathbf{u}|^{2}|\mathbf{u}| d x d t \\
& \leq c \int_{Q_{T^{\prime}}}|\nabla \mathbf{u}|^{3} d x d t+\left(T^{\prime}\right)^{\frac{q-2}{2 q}} \mathcal{K}^{\frac{2}{q}}\left\|\left|\mathbf{u}\|\nabla \mathbf{u} \mid\|_{2 ; Q_{T^{\prime}}}\right.\right.
\end{aligned}
$$

For the exterior force term,

$$
\left|\int_{Q_{T^{\prime}}} \mathbf{f}_{y_{\gamma}}: \mathbf{u}_{y_{\gamma}} d y d t\right| \leq c \int_{Q_{T^{\prime}}}|\nabla \mathbf{u}|^{3} d x d t+c \int_{Q_{T^{\prime}}}|\nabla \mathbf{f}|^{\frac{3}{2}} d x d t .
$$

Inserting these estimates into (2.16) and (2.17) respectively we obtain

$$
\begin{aligned}
\|\nabla \sigma\|_{q^{\prime} ; Q_{T^{\prime}}}^{2} \leq & 2 \mathcal{K}^{\frac{4(q-1)}{q}}+c\left(T^{\prime}\right)^{\frac{q-2}{q}} \int_{Q_{T^{\prime}}}|\mathbf{u}|^{2}|\nabla \mathbf{u}|^{2} d x d t \\
& +\mathcal{K}^{\frac{2(q-2)}{q}} \int_{Q_{T^{\prime}}}|\nabla \mathbf{u}|^{3} d x d t+\mathcal{K}^{\frac{2(q-2)}{q}}\|\nabla \mathbf{f}\|_{\frac{3}{2}, Q_{T^{\prime}}}^{\frac{3}{2}}
\end{aligned}
$$

and

$$
\begin{aligned}
\int_{Q_{T^{\prime}}}|\mathbf{D}(\mathbf{u})|^{q-2}|\nabla \mathbf{D}(\mathbf{u})|^{2} d y d t \leq & \mathcal{K}^{2}+\mathcal{K}^{\frac{4}{q}}+c\left(T^{\prime}\right)^{\frac{q-2}{q}} \int_{Q_{T^{\prime}}}|\mathbf{u}|^{2}|\nabla \mathbf{u}|^{2} d x d t \\
& +c \int_{Q_{T^{\prime}}}|\nabla \mathbf{u}|^{3} d x d t+\|\nabla \mathbf{f}\|_{\frac{3}{2}, Q_{T^{\prime}}}^{\frac{3}{2}} \equiv \mathcal{M}\left(T^{\prime}\right) .
\end{aligned}
$$

We use the following notations

$$
\begin{aligned}
\mathcal{J}\left(T^{\prime}\right) & \equiv\|\nabla \mathbf{u}\|_{q, 3 q: Q_{T^{\prime}}}^{q}, \\
\mathcal{E}\left(T^{\prime}\right) & \equiv c\left(\mathcal{K}^{2}+\|\nabla \mathbf{u}\|_{\infty, q ; Q_{T^{\prime}}}^{q}+\|\mid \mathbf{u}\| \nabla \mathbf{u} \|_{2 ; Q_{T^{\prime}}}^{2}\right) .
\end{aligned}
$$

Clearly,

$\int_{0}^{T^{\prime}}\left(\int_{\Omega}|\mathbf{D} \mathbf{u}|^{3 q} d y\right)^{\frac{1}{3}} d t \leq\left.\left.\int_{Q_{T^{\prime}}}|\nabla| \mathbf{D} \mathbf{u}\right|^{\frac{q}{2}}\right|^{2} \leq \frac{q^{2}}{4} \int_{Q_{T^{\prime}}}|\mathbf{D}(\mathbf{u})|^{q-2}|\nabla \mathbf{D}(\mathbf{u})|^{2} d y d t$. 
Thus, using Korn's inequality and taking into account (2.20) get

$$
\mathcal{J}\left(T^{\prime}\right) \leq c \mathcal{M}\left(T^{\prime}\right) .
$$

Applying Hölder's and Young's inequalities one finds

$$
\begin{aligned}
\|\nabla \mathbf{u}\|_{3 ; Q_{T^{\prime}}}^{3} & \leq\left(T^{\prime}\right)^{\frac{5 q-9}{2 q}}\|\nabla \mathbf{u}\|_{\infty}^{\frac{3 q-3}{2}}, \| \nabla ; Q_{T^{\prime}} \\
& \leq\left(T^{\prime}\right)^{\frac{5 q-9}{2 q}} \mathcal{E} \|_{T^{\prime}\left(T^{\prime}\right)^{\frac{3 q-3}{2 q}}}^{\frac{9-3 q}{2}} \mathcal{J}\left(T_{T^{\prime}}\right)^{\frac{9-3 q}{2 q}} \\
& \leq T^{\prime} \mathcal{E}\left(T^{\prime}\right)^{\frac{3 q-3}{5 q-9}}+\delta \mathcal{J}\left(T^{\prime}\right)
\end{aligned}
$$

for small number $\delta$. Thus, we obtain

$$
\mathcal{J}\left(T^{\prime}\right) \leq c \mathcal{M}\left(T^{\prime}\right) \leq\left(T^{\prime}\right)^{\frac{q-2}{q}} \mathcal{E}\left(T^{\prime}\right)+T^{\prime} \mathcal{E}\left(T^{\prime}\right)^{\frac{3 q-3}{5 q-9}}+\mathcal{K}^{2}+\|\nabla \mathbf{f}\|_{\frac{3}{2}, Q_{T^{\prime}}}^{\frac{3}{2}} .
$$

For simplicity, we use the notation $\mathcal{K}_{1}^{2} \equiv \mathcal{K}^{2}+\|\nabla \mathbf{f}\|_{\frac{3}{2}, Q_{T^{\prime}}}^{\frac{3}{2}}$.

According to Lemma 2.1 we deduce

$$
\mathcal{E}\left(T^{\prime}\right) \leq c\left(\mathcal{K}^{2}+\mathcal{K}^{4 q \rho}\left(T^{\prime}\right)^{(7 q-12) v} \mathcal{J}\left(T^{\prime}\right)^{3(4-q) v}\right) .
$$

Into the right of (2.23) inserting (2.22) we obtain

$$
\begin{aligned}
\mathcal{E}\left(T^{\prime}\right) \leq c \mathcal{K}^{4 q \rho} & \left(\left(T^{\prime}\right)^{\frac{2 v\left(2 q^{2}+3 q-12\right)}{q}} \mathcal{E}\left(T^{\prime}\right)^{\frac{-4\left(5 q^{2}-25 q+24\right)}{(5 q+12)(q-2)}}\right. \\
& \left.+\left(T^{\prime}\right)^{4 q v} \mathcal{E}\left(T^{\prime}\right)^{\frac{2 q(35 q-52)(3-q)}{(5 q-9)(5 q+12)(q-2)}}\right) \mathcal{E}\left(T^{\prime}\right) \\
+ & \left(\mathcal{K}_{1}^{\frac{-2 q^{2}+108 q-144}{(5 q+12)(q-2)}}\left(T^{\prime}\right)^{(7 q-12) v}+\mathcal{K}^{2}\right) \\
\equiv & \Psi\left(T^{\prime}\right) \mathcal{E}\left(T^{\prime}\right)+c\left(\mathcal{K}_{1}^{\frac{-2 q^{2}+108 q-144}{(5 q+12)(q-2)}}\left(T^{\prime}\right)^{(7 q-12) v}+\mathcal{K}^{2}\right) .
\end{aligned}
$$

Clearly, $\Psi$ is an increasing and continuous function with $\Psi(0)=0$. In case $\Psi(T) \leq \frac{1}{2}$ we put $T_{*}=T$ and there holds

$$
\mathcal{E}(T) \leq 2 c\left(\mathcal{K}_{1}^{\frac{-2 q^{2}+108 q-144}{(5 q+12)(q-2)}} T^{(7 q-12) v}+\mathcal{K}^{2}\right) \equiv \mathcal{K}_{2} .
$$

Otherwise, there exists $T_{*} \in(0, T)$, such that

$$
\Psi\left(T_{*}\right)=\frac{1}{2} .
$$

Thus,

$$
\mathcal{E}\left(T_{*}\right) \leq 2 c\left(\mathcal{K}_{1}^{\frac{-2 q^{2}+108 q-144}{(5 q+12)(q-2)}} T_{*}^{(7 q-12) v}+\mathcal{K}^{2}\right) \leq \mathcal{K}_{2} .
$$

In case $T_{*} \leq 1$, we have

$$
\begin{aligned}
\frac{1}{2} & =\Psi\left(T_{*}\right) \\
& \leq c \mathcal{K}^{4 q \rho} T_{*}^{\frac{2 v\left(2 q^{2}+3 q-12\right)}{q}}\left(\mathcal{K}_{2}^{\frac{-4\left(5 q^{2}-25 q+24\right)}{(5 q+12)(q-2)}}+\mathcal{K}_{2}^{\frac{2 q(35 q-52)(3-q)}{(5 q-9)(5 q+12)(q-2)}}\right)
\end{aligned}
$$


since

$$
\frac{2 v\left(2 q^{2}+3 q-12\right)}{q} \leq 4 q v .
$$

This implies

$$
T_{*} \geq\left[2 c \mathcal{K}^{4 q \rho}\left(\mathcal{K}_{2}^{\frac{-4\left(5 q^{2}-25 q+24\right)}{(5 q+12)(q-2)}}+\mathcal{K}_{2}^{\frac{2 q(35 q-52)(3-q)}{(5 q-9)(5 q+12)(q-2)}}\right)\right]^{-\frac{q(5 q+12)(q-2)}{2(5 q-6)\left(2 q^{2}+3 q-12\right)}} .
$$

On the contrary, if $T_{*}>1$ we get

$$
T_{*} \geq\left[2 c \mathcal{K}^{4 q \rho}\left(\mathcal{K}_{2}^{\frac{-4\left(55^{2}-25 q+24\right)}{(5 q+12)(q-2)}}+\mathcal{K}_{2}^{\frac{2 q(35 q-52)(3-q)}{(5 q-9)(5 q+12)(q-2)}}\right)\right]^{-\frac{(5 q+12)(q-2)}{4 q(5 q-6)}} .
$$

Thus, we have the following result.

Theorem 2.2. Let $\left(\mathbf{u}_{\varepsilon}, \nabla p_{\varepsilon}\right)$ be a solution to the approximate system (2.1). Then

$$
\left\|\mathbf{u}_{\varepsilon, t}\right\|_{2 ; Q_{T_{0}}}+\left\|\nabla \mathbf{u}_{\varepsilon}\right\|_{\infty, q ; Q_{T_{0}}}+\left\|\nabla \mathbf{u}_{\varepsilon}\right\|_{q, 3 q ; Q_{T_{0}}} \leq c \mathcal{K}_{2}
$$

for all $0<\varepsilon<1$, where $T_{0}$ is the minimum of (2.24) and (2.25). Furthermore, we have from (2.19), (2.20) and (2.21) that

$$
\begin{aligned}
\left\|\nabla \mathbf{u}_{\varepsilon}\right\|_{3 ; Q_{T_{0}}} & \leq c \mathcal{K}_{2}, \\
\int_{Q_{T_{0}}}\left|\mathbf{D}\left(\mathbf{u}_{\varepsilon}\right)\right|^{q-2}|\nabla \mathbf{D}(\mathbf{u})|^{2} d y d t & \leq c \mathcal{K}_{2}, \\
\left\|\nabla \sigma_{\varepsilon}\right\|_{q^{\prime} ; Q_{T^{\prime}}} & \leq c \mathcal{K}_{2},
\end{aligned}
$$

where $c$ is independent of $\varepsilon$.

\section{A regularity criteroin and time singularity}

In this section, we first provide a regularity criterion like the Serrin condition for the Navier-Stokes fluid, and then estimate the Hausdorff dimension of the set of singular times.

Proof of Theorem 1.4. Like (2.10) taking $\varphi=-\Delta^{-h} \Delta^{h} \mathbf{u}$ as a test function and considering the divergence free property, we obtain

$$
\begin{aligned}
\frac{1}{2} & \frac{d}{d t}\left\|\Delta^{h} \mathbf{u}\right\|_{2 ; \Omega}^{2}+\int_{\Omega} \Delta^{h} \sigma: \Delta^{h} \mathbf{D} \mathbf{u} d y \\
\quad & =-\int_{\Omega}\left(\left(\Delta^{h} \mathbf{u}\right) \cdot \nabla \mathbf{u}\right) \cdot \Delta^{h} \mathbf{u} d y d t+\int_{\Omega} \Delta^{h} \mathbf{f} \cdot \Delta^{h} \mathbf{u} d y d t .
\end{aligned}
$$

This yields

$$
\begin{aligned}
& \frac{1}{2} \frac{d}{d t}\left\|\Delta^{h} \mathbf{u}\right\|_{2 ; \Omega}^{2}+\int_{\Omega}\left|\mathbf{D}(\mathbf{u})+\mathbf{D}\left(\mathbf{u}\left(\cdot+h \mathbf{e}_{\gamma}\right)\right)\right|^{q-2}\left|\Delta^{h} \mathbf{D}(\mathbf{u})\right|^{2} d y \\
& \quad \leq \int_{\Omega}\left|\Delta^{h} \mathbf{u}\right|^{2}|\nabla \mathbf{u}| d y+\int_{\Omega}\left|\Delta^{h} \mathbf{f}\right|\left|\Delta^{h} \mathbf{u}\right| d y .
\end{aligned}
$$


On the other hand,

$$
\begin{aligned}
\left\|\Delta^{h} \sigma\right\|_{q^{\prime} ; Q_{T}}^{2} \leq & c\left(\int_{Q_{T}}\left|\mathbf{D}(\mathbf{u})+\mathbf{D}\left(\mathbf{u}\left(\cdot+h \mathbf{e}_{\gamma}\right)\right)\right|^{(q-2) q^{\prime}}\left|\Delta^{h} \mathbf{D}(\mathbf{u})\right|^{q^{\prime}} d y d t\right)^{\frac{2}{q^{\prime}}} \\
\leq & c\left(\int_{Q_{T}}\left|\mathbf{D}(\mathbf{u})+\mathbf{D}\left(\mathbf{u}\left(\cdot+h \mathbf{e}_{\gamma}\right)\right)\right|^{\frac{(q-2) q^{\prime}}{2} \frac{2}{2-q^{\prime}}} d y d t\right)^{\frac{2}{q^{\prime}} \frac{2-q^{\prime}}{2}} \\
& \times\left(\int_{Q_{T}}\left|\mathbf{D}(\mathbf{u})+\mathbf{D}\left(\mathbf{u}\left(\cdot+h \mathbf{e}_{\gamma}\right)\right)\right|^{q-2}\left|\Delta^{h} \mathbf{D}(\mathbf{u})\right|^{2} d y d t\right)
\end{aligned}
$$

and

$$
\begin{aligned}
\left\|\Delta^{h} \sigma\right\|_{q^{\prime} ; Q_{T^{\prime}}}^{2} \leq & \mathcal{K}^{\frac{2(q-2)}{q}}\left\|\nabla \mathbf{u}_{0}\right\|_{2}^{2}+\mathcal{K}^{\frac{2(q-2)}{q}}\left|\int_{Q_{T^{\prime}}} \Delta^{h} \mathbf{h}_{\varepsilon}: \Delta^{h} \mathbf{u} d y d t\right| \\
& +\mathcal{K}^{\frac{2(q-2)}{q}}\left|\int_{Q_{T^{\prime}}} \Delta^{h} \mathbf{f}: \Delta^{h} \mathbf{u} d y d t\right|
\end{aligned}
$$

Now, passing to the limit, we obtain

$$
\frac{d}{d t}\|\nabla \mathbf{u}\|_{2 ; \Omega}^{2}+2 \int_{\Omega}|\mathbf{D}(\mathbf{u})|^{q-2}|\nabla \mathbf{D}(\mathbf{u})|^{2} d y \leq \int_{\Omega}|\nabla \mathbf{u}|^{3} d y+\int_{\Omega}|\nabla \mathbf{f}||\nabla \mathbf{u}| d y
$$

and therefore, we have

$$
\frac{d}{d t}\|\nabla \mathbf{u}\|_{2 ; \Omega}^{2}+2 \int_{\Omega}|\mathbf{D}(\mathbf{u})|^{3 q} d y \leq \int_{\Omega}|\nabla \mathbf{u}|^{3} d y+\int_{\Omega}|\nabla \mathbf{f}||\nabla \mathbf{u}| d y .
$$

On the other hand, we observe that

$$
\|\nabla \sigma\|_{q^{\prime} ; Q_{T}}^{2} \leq \mathcal{K}^{\frac{2(q-2)}{q}}\left(\mathcal{K}^{2}+\int_{Q_{T}}|\nabla \mathbf{u}|^{3} d x d t+\|\nabla \mathbf{f}\|_{\frac{3}{2}, Q_{T}}^{\frac{3}{2}}\right)
$$

and

$$
\int_{Q_{T}}|\mathbf{D}(\mathbf{u})|^{q-2}|\nabla \mathbf{D}(\mathbf{u})|^{2} d y d t \leq \mathcal{K}^{2}+c \int_{Q_{T}}|\nabla \mathbf{u}|^{3} d x d t+\|\nabla \mathbf{f}\|_{\frac{3}{2}, Q_{T}}^{\frac{3}{2}} .
$$

We use the following notations

$$
\begin{aligned}
\mathcal{J}(T) & \equiv\|\nabla \mathbf{u}\|_{q, 3 q: Q_{T}}^{q}, \\
\mathcal{E}(T) & \equiv c\left(\mathcal{K}^{2}+\|\nabla \mathbf{u}\|_{\infty, q ; Q_{T}}^{q}+\||\mathbf{u}| \mid \nabla \mathbf{u}\|_{2 ; Q_{T}}^{2}\right) .
\end{aligned}
$$

By the Young and interpolation inequalities, we obtain

$$
\|\nabla \mathbf{u}\|_{3 ; \Omega}^{3} \leq\|\nabla \mathbf{u}\|_{\frac{\frac{6(\alpha+q-3)}{2 \alpha+3 q-7} ; \Omega}{2 \alpha+6}}^{\frac{6(\alpha+q-3)}{2 \alpha+3}}\|\nabla \mathbf{u}\|_{3 q ; \Omega}^{\frac{3 q}{2 \alpha+3 q-6}} \leq c\|\nabla \mathbf{u}\|_{\frac{\frac{6(\alpha+q-3)}{2 \alpha+3 q-7} ; \Omega}{2 \alpha+9}}^{\frac{6(\alpha+q-3)}{2 \alpha+3}}+\epsilon\|\nabla \mathbf{u}\|_{3 q ; \Omega}^{q} .
$$

Using the interpolation inequality again, we have

$$
\|\nabla \mathbf{u}\|_{\frac{6(\alpha+q-3)}{2 \alpha+3 q-7} ; \Omega}^{\frac{6(\alpha+q-3)}{2 \alpha+3 q-9}} \leq\|\nabla \mathbf{u}\|_{2 ; \Omega}^{2}\|\nabla \mathbf{u}\|_{\alpha ; \Omega}^{\frac{2 \alpha}{2 \alpha+3 q-9}}
$$


Thus, we obtain

$$
\begin{aligned}
\mathcal{J}(T) & \leq \mathcal{K}^{2}+c \int_{0}^{T}\|\nabla \mathbf{u}\|_{2 ; \Omega}^{2}\|\nabla \mathbf{u}\|_{\alpha ; \Omega}^{\frac{2 \alpha}{2 \alpha+3 q-9}} d t+\|\nabla \mathbf{f}\|_{\frac{3}{2}, Q_{T}}^{\frac{3}{2}} \\
& \leq \mathcal{K}^{2}+c \sup _{t}\|\nabla \mathbf{u}\|_{2 ; \Omega}^{2}\|\nabla \mathbf{u}\|_{L_{t, x}^{\beta, \alpha}\left(Q_{T}\right)}+\|\nabla \mathbf{f}\|_{\frac{3}{2}, Q_{T}}^{\frac{3}{2}} .
\end{aligned}
$$

According to Lemma 2.1 we deduce

$$
\mathcal{E}\left(T^{\prime}\right) \leq c\left(\mathcal{K}^{2}+\mathcal{K}^{4 q \rho}\left(T^{\prime}\right)^{(7 q-12) v} \mathcal{J}\left(T^{\prime}\right)^{\frac{(12-3 q)(5 q-6)}{(5 q+12)(q-2)}}\right)
$$

Into the right of (3.2) inserting (2.22) we obtain

$$
\begin{gathered}
\mathcal{E}\left(T^{\prime}\right) \leq c \mathcal{K}^{4 q \rho}\left(\left(T^{\prime}\right)^{\frac{2 v\left(2 q^{2}+3 q-12\right)}{q}} \mathcal{E}\left(T^{\prime}\right)^{\frac{-4\left(5 q^{2}-25 q+24\right)}{(5 q+12)(q-2)}}\right. \\
\left.+\left(T^{\prime}\right)^{4 q v} \mathcal{E}\left(T^{\prime}\right)^{\frac{2 q(35 q-52)(3-q)}{(5 q-9)(5 q+12)(q-2)}}\right) \mathcal{E}\left(T^{\prime}\right) \\
+c\left(\mathcal{K}_{1}^{\frac{-2 q^{2}+108 q-144}{(5 q+12)(q-2)}}\left(T^{\prime}\right)^{(7 q-12) v}+\mathcal{K}^{2}\right) \\
\equiv \Psi\left(T^{\prime}\right) \mathcal{E}\left(T^{\prime}\right)+c\left(\mathcal{K}_{1}^{\frac{-2 q^{2}+108 q-144}{(5 q+12)(q-2)}}\left(T^{\prime}\right)^{(7 q-12) v}+\mathcal{K}^{2}\right) .
\end{gathered}
$$

Clearly, $\Psi$ is an increasing and continuous function with $\Psi(0)=0$. In case $\Psi(T) \leq \frac{1}{2}$ we put $T_{*}=T$ and there holds

$$
\mathcal{E}(T) \leq 2 c\left(\mathcal{K}_{1}^{\frac{-2 q^{2}+108 q-144}{(5 q+12)(q-2)}} T^{(7 q-12) v}+\mathcal{K}^{2}\right) \equiv \mathcal{K}_{2} .
$$

This estimate implies via following similar methods of Theorem 2.2 that $u$ is a strong solution to (1.2). We complete the proof.

We now estimate the Hausdorff dimension of the set of singular times. The argument is similar to Theorem 4.2 in [3]. In [3] the exterior force term $\mathbf{f}$ is independent of time $t$, but with this assumption there was a little error in the proof, which can be fixed easily if we add the dependency of $\mathbf{f}$ on time $t$. For the reader's convenience, we present the whole estimates for the proof.

Proof of Theorem 1.5. We note that

$$
\begin{aligned}
& \int_{\Omega}|\nabla \mathbf{u}|^{3} d x=\int_{\Omega}|\nabla \mathbf{u}|^{(3-q)+\frac{q(3 q-5)}{4}+\frac{3 q(3-q)}{4}} d x \\
& \leq\left(\int_{\Omega}|\nabla \mathbf{u}|^{2} d x\right)^{\frac{3-q}{2}}\left(\int_{\Omega}|\nabla \mathbf{u}|^{q} d x\right)^{\frac{3 q-5}{4}}\left(\int_{\Omega}|\nabla \mathbf{u}|^{3 q} d x\right)^{\frac{3-q}{4}} \\
& \leq \frac{C}{\epsilon}\left(\int_{\Omega}|\nabla \mathbf{u}|^{2} d x\right)^{\frac{6-2 q}{3 q-5}} \int_{\Omega}|\nabla \mathbf{u}|^{q} d x+\epsilon\left(\int_{\Omega}|\nabla \mathbf{u}|^{3 q} d x\right)^{\frac{1}{3}} .
\end{aligned}
$$

Set

$$
A \equiv 1+\|\nabla \mathbf{u}\|_{2}^{2}
$$


Considering the inner product of (1.2) with

$$
\frac{-\Delta^{-h} \Delta^{h} \mathbf{u}}{A^{\frac{6-2 q}{3 q-5}}}
$$

using the difference quotient method as in the process obtaining (3.1) and taking $\epsilon$ small, we have

$$
\begin{array}{r}
\frac{3 q-5}{2(5 q-11)} \frac{d}{d t} A^{\frac{5 q-11}{3 q-5}}+\frac{C}{A^{\frac{6-2 q}{3 q-5}}} \int_{\Omega}|\mathbf{D}(u)|^{q-2}|\nabla \mathbf{D}(u)|^{2} d x \\
\leq C \int_{\Omega}|\nabla \mathbf{u}|^{q} d x+\frac{C}{A^{\frac{6-2 q}{3 q-5}}} \int_{\Omega}|\nabla \mathbf{f}|^{q^{\prime}} d x .
\end{array}
$$

and

$$
\frac{3 q-5}{2(5 q-11)} \frac{d}{d t} A^{\frac{5 q-11}{3 q-5}} \leq C \int_{\Omega}|\nabla \mathbf{u}|^{q} d x+\frac{C}{A^{\frac{6-2 q}{3 q-5}}} \int_{\Omega}|\nabla \mathbf{f}|^{q^{\prime}} d x .
$$

Let $S$ be a singular time. Integrating from $s$ to $S$, we obtain

$$
\begin{aligned}
\frac{3 q-5}{2(11-5 q)}\left[A^{\frac{5 q-11}{3 q-5}}(s)-A^{\frac{5 q-11}{3 q-5}}(S)\right] \leq & C \int_{s}^{S} \int_{\Omega}|\nabla \mathbf{u}|^{q} d x d t \\
& +C \int_{s}^{S} \int_{\Omega}|\nabla \mathbf{f}|^{q^{\prime}} d x d t .
\end{aligned}
$$

Since $S$ is a singular time, with the aid of Theorem 1.4, we note that

$$
\int_{\Omega}|\nabla \mathbf{u}|^{2}(S) d x=\infty
$$

and therefore, $A^{\frac{5 q-11}{3 q-5}}(S)=0$. Hence we have

$$
\begin{aligned}
\frac{3 q-5}{2(11-5 q)}\left(\int_{\Omega}|\nabla \mathbf{u}(s)|^{2} d x\right)^{\frac{5 q-11}{3 q-5}} \leq & C \int_{s}^{S} \int_{\Omega}|\nabla \mathbf{u}|^{q} d x d t \\
& +C \int_{s}^{S} \int_{\Omega}|\nabla \mathbf{f}|^{q^{\prime}} d x d t .
\end{aligned}
$$

By Hölder's inequality, we have

$$
\left(\int_{\Omega}|\nabla \mathbf{u}(s)|^{q} d x\right)^{\frac{2(5 q-11)}{q(3 q-5)}} \leq C \int_{s}^{S} \int_{\Omega}|\nabla \mathbf{u}|^{q} d x d t+C \int_{s}^{S} \int_{\Omega}|\nabla \mathbf{f}|^{q^{\prime}} d x d t .
$$

Setting

$$
U(s) \equiv \int_{s}^{S} \int_{\Omega}|\nabla \mathbf{u}(\tau)|^{q} d x d \tau
$$

and

$$
F(s) \equiv \int_{s}^{S} \int_{\Omega}|\nabla \mathbf{f}(\tau)|^{q^{\prime}} d x d \tau
$$

we have

$$
\int_{\Omega}|\nabla \mathbf{u}(t)|^{q} d x=-U^{\prime}(t)
$$


and

$$
\int_{\Omega}|\nabla \mathbf{f}(t)|^{q^{\prime}} d x=-F^{\prime}(t) .
$$

Thus, we obtain

$$
\left(-U^{\prime}(t)\right)^{\frac{2(5 q-11)}{q(3 q-5)}} \leq C U(t)+C F(t),
$$

and

$$
\left(-U^{\prime}(t)\right)^{\frac{2(11-5 q)}{q(3 q-5)}} \geq \frac{C}{U(t)+F(t)} .
$$

Therefore, we have

$$
-U^{\prime}(t)(U(t)+F(t))^{\frac{q(3 q-5)}{2(11-5 q)}} \geq C .
$$

Since $-F^{\prime}(t) \geq 0$, we have

$$
\left(-U^{\prime}(t)-F^{\prime}(t)\right)(U(t)+F(t))^{\frac{q(3 q-5)}{2(11-5 q)}} \geq C,
$$

and

$$
\left(U^{\prime}(t)+F^{\prime}(t)\right)(U(t)+F(t))^{\frac{q(3 q-5)}{2(11-5 q)}} \leq-C .
$$

Integrating from $s$ to $S$, we have

$$
(U(S)+F(S))^{\frac{22-15 q+3 q^{2}}{2(11-5 q)}}-(U(s)+F(s))^{\frac{22-15 q+3 q^{2}}{2(11-5 q)}} \leq-C(S-s) .
$$

Since $U(S)=F(S)=0$, we have

$$
\int_{S}^{S} \int_{\Omega}|\nabla \mathbf{u}|^{q} d x d t+\int_{s}^{S} \int_{\Omega}|\nabla \mathbf{f}|^{q^{\prime}} d x d t \geq C(S-s)^{\frac{2(11-5 q)}{22-15 q+3 q^{2}}}
$$

Let

$$
\mathcal{O} \equiv\left\{t: \int_{\Omega}|\nabla \mathbf{u}|^{2} d x<\infty\right\}
$$

then $\mathcal{O}$ is right open. So $\mathcal{O}$ is the countable union of semi-open intervals, say,

$$
\mathcal{O}=\bigcup\left[a_{i}, b_{i}\right)
$$

In particular, we set the open set

$$
\mathcal{O}_{1}=\bigcup\left(a_{i}, b_{i}\right)
$$

then $\mathcal{S} \equiv[0, T] \backslash \mathcal{O}_{1}$ is closed and has Lebesgue measure zero. Let $t \in\left(a_{i}, b_{i}\right)$. Since we have local existence of the strong solution, we obtain

$$
\sum\left(b_{i}-a_{i}\right)^{\frac{2(11-5 q)}{22-15 q+3 q^{2}}} \leq C \int_{0}^{T} \int_{\Omega}|\nabla \mathbf{u}|^{q} d x d t+C \int_{0}^{T} \int_{\Omega}|\nabla \mathbf{f}|^{q^{\prime}} d x d t<\infty .
$$

For every $\epsilon>0$, we can choose a finite part $I_{\epsilon}$ of $I$ satisfying

$$
\sum_{i \notin I_{\epsilon}}\left(b_{i}-a_{i}\right) \leq \epsilon, \quad \sum_{i \notin I_{\epsilon}}\left(b_{i}-a_{i}\right)^{\frac{2(11-5 q)}{22-15 q+3 q^{2}}} \leq \epsilon .
$$


The set $[0, T] \backslash \bigcup_{i \in I_{\epsilon}}\left(a_{i}, b_{i}\right)$ is the union of finite number of mutually disjoint closed intervals $B_{j}, j=1,2, \ldots, N$. It is clear that $\bigcup_{j=1}^{N} B_{j} \supset \mathcal{S}$. Since $\left(a_{i}, b_{i}\right)$ are mutually disjoint, $\left(a_{i}, b_{i}\right)$ is contained in one and only one interval $B_{i}$. We denote $I_{j}$ the set of $i$ 's satisfying $B_{j} \supset\left(a_{i}, b_{i}\right)$. It is clear that

$$
B_{j}=\left(\bigcup_{i \in I_{j}}\left(a_{i}, b_{i}\right)\right) \bigcup\left(B_{j} \cap \mathcal{S}\right), \quad \text { for all } j .
$$

Hence we have

$$
\operatorname{diam} B_{j}=\sum\left(b_{i}-a_{i}\right) \leq \epsilon,
$$

and

$$
\begin{aligned}
\left(d H^{\frac{2(11-5 q)}{22-15 q+3 q^{2}}}\right)(\mathcal{S}) & \leq \sum_{j=1}^{N}\left(\operatorname{diam} B_{j}\right)^{\frac{2(11-5 q)}{22-15 q+3 q^{2}}} \\
& \leq \sum_{j=1}^{N}\left(\sum_{i \in I_{j}}\left(b_{i}-a_{i}\right)\right)^{\frac{2(11-5 q)}{22-15 q+3 q^{2}}} \\
& \leq \sum_{i \notin I_{\epsilon}}\left(b_{i}-a_{i}\right)^{\frac{2(11-5 q)}{2^{2-15 q+3 q^{2}}}} \leq \epsilon
\end{aligned}
$$

where $d H^{k}$ is the $k$-dimensional Hausdorff measure. Since $\epsilon$ is chosen arbitrary, we complete the proof.

\section{Acknowledgments}

The authors thank to the editor and reviewers for careful reading and helpful comments.

\section{References}

[1] Bae, H.-O., Choe, H.J.: $L^{\infty}$-bound of weak solutions to Navier-Stokes equations. In: Proceedings of the Korea-Japan Partial Differential Equations Conference (Taejon, 1996). Lecture Notes Ser. 39. Seoul Nat. Univ., Seoul, p. 13 (1997)

[2] Bae, H.-O., Choe, H.J.: A regularity criterion for the Navier-Stokes equations. Comm. Partial Differ. Equ. 32, 1173-1187 (2007)

[3] Bae, H.-O., Choe, H.J.: Existence of weak solutions to a class of non-newtonian flows. Houst. J. Math. 26, 387-408 (2000)

[4] Bae, H.-O., Choe, H.J., Kim, D.W.: Regularity and singularity of weak solutions to Ostwald-de Waele flows. International Conference on Differential Equations and Related Topics (Pusan, 1999). J. Korean Math. Soc. 37(6), 957-975 (2000) 
[5] da Veiga, H.B.: On the smoothness of a class of weak solutions to the NavierStokes equations. J. Math. Fluid Mech. 2, 315-323 (2000)

[6] Berselli, L.C., Diening, L., Ruzicka, M.: Existence of strong solutions for incompressible fluids with shear dependent viscosities. J. Math. Fluid Mech. 12, 101$132(2010)$

[7] Chae, D., Choe, H.J.: Regularity of solutions to the Navier-Stokes equation. Electron. J. Differ. Equ. 5, 1-7 (1999)

[8] Chae, D., Lee, J.: Regularity criterion in terms of pressure for the Navier-Stokes equations. Nonlinear Anal. 46(5), Ser. A: Theory Methods, 727-735 (2001)

[9] Diening, L., Ruzicka, M.: Strong solutions for generalized Newtonian fluids. J. Math. Fluid Mech. 7, 413-450 (2005)

[10] Diening, L., Ruzicka, M., Wolf, J.: Existence of weak solutions for unsteady motions of generalized Newtonian fluids. Ann. Sc. Norm. Super. Pisa Cl. Sci. (5) 9(1), 1-46 (2010)

[11] Fabes, E.B., Jones, B.F., Riviere, N.M.: The initial value problem for the NavierStokes equations with data in $L^{p}$. Arch. Ration. Mech. Anal. 45, 222-240 (1972)

[12] Gala, S.: Regularity criterion on weak solutions to the Navier-Stokes equations. J. Korean Math. Soc. 45, 537-558 (2008)

[13] Kukavica, I., Ziane, M.: One component regularity for the Navier-Stokes equations. Nonlinearity 19(2), 453-469 (2006)

[14] Ladyzhenskaya, O.A.: The Mathematical Theory of Viscous Incompressible Flow, 2nd edn. Gordon and Breach, New York (1969)

[15] Málek, J., Nečas, J., Rokyta, M., Ružička, M.: Weak and Measure-valued Solutions to Evolutionary PDEs. Chapman \& Hall (1996)

[16] Malek, J., Necas, J., Ruzicka, M.: On weak solutions to a class of non-Newtonian incompressible fluids in bounded three-dimensional domains: the case $p \geq 2$. Adv. Differ. Equ. 6(3), 257-302 (2001)

[17] Neustupa, J., Novotny, A., Penel, P.: An interior regularity of a weak solution to the Navier-Stokes equations in dependence on one component of velocity. Top. Math. Fluid Mech. Quad. Mat. 10, 163-183 (2002)

[18] Pokorny, M.: Cauchy problem for the non-Newtonian viscous incompressible fluid. Appl. Math. 41(3), 169-201 (1996)

[19] Prodi, G.: Un teorema di unicit per le equazioni di Navier-Stokes. Ann. Mat. Pura Appl. 48(4), 173-182 (Italian) (1959)

[20] Serrin, J.: On the interior regularity of weak solutions of the Navier Stokes equations. Arch. Ration. Mech. Anal. 9, 187-195 (1962)

[21] Wolf, J.: Existence of weak solutions to the equations of non-stationary motion of non-Newtonian fluids with shear rate dependent viscosity. J. Math. Fluid Mech. 9(1), 104-138 (2007) 
[22] Zhou, Y.: A new regularity criterion for the Navier-Stokes equations in terms of the gradient of one velocity component. Methods Appl. Anal. 9(4), 563$578(2002)$

Hyeong-Ohk Bae

Department of Financial Engineering

Ajou University

Suwon

Republic of Korea

e-mail: hobae@ajou.ac.kr

Kyungkeun Kang

Department of Mathematics

Yonsei University

Seoul

Korea

e-mail: kkang@yonsei.ac.kr

Jihoon Lee

Department of Mathematics

Chung-Ang University

Seoul

Korea

e-mail: jhlee.pde@gmail.com

Jörg Wolf

Department of Mathematics

Humboldt-University of Berlin

Unter den Linden 6

10099 Berlin

Germany

e-mail: jwolf@math.hu-berlin.de

Received: 14 November 2013.

Accepted: 10 March 2014. 